\title{
0-1 Laws of a Probability Measure on a Locally Convex Space
}

\author{
By \\ Yasuji TAKAHASH* and Yoshiaki OKAZAKI**
}

\begin{abstract}
We introduce several 0-1 laws for a cylindrical probability measure $\mu$ on a locally convex Hausdorff space $E$ and examine the equivalence of them. We show that the following 0-1 laws are equivalent: (a) for every $x_{n}^{\prime}$ in $E^{\prime}, \mu\left(x ;\left(x_{n}^{\prime}(x)\right) \in c_{0}\right)=0$ or 1 , (b) for every $x_{n}^{\prime}$ in $E^{\prime}, \mu\left(x ;\left(x_{n}^{\prime}(x)\right) \in c_{1}\right)=0$ or 1 , and (c) for every $x_{n}^{\prime}$ in $E^{\prime}$. $\mu\left(x ;\left(x_{n}^{\prime}(x)\right) \in l_{\infty}\right)=0$ or 1 . We also show that each of (a), (b) and (c) implies: (d) for every $x_{n}^{\prime}$ in $E^{\prime}, \mu\left(x ;\left(x_{n}^{\prime}(x)\right) \in l_{p}\right)=0$ or 1 . If $\mu$ is a Radon probability measure, then (a), (b) and (c) are equivalent to: (e) for every lower semi-continuous semi-norm $N$. $\mu(x ; N(x)<\infty)=0$ or 1 .
\end{abstract}

\section{$\S 1.0-1$ Laws}

In this section, we present several 0-1 laws which appear in the probability theory.

Let $E$ be a locally convex Hausdorff space, $C\left(E, E^{\prime}\right)$ be the cylindrical $\sigma$-algebra generated by the topological dual $E^{\prime}$ and $\mathcal{B}(E)$ be the Borel $\sigma$-algebra generated by all open subsets. Let $\mu$ be a probability measure on $C\left(E, E^{\prime}\right)$ or on $\mathscr{B}(E)$. The measure $\mu$ on $\mathscr{B}(E)$ is called a Radon measure if it holds $\mu(A)$ $=\sup \{\mu(K) ; K \subset A$ and $K$ is compact $\}$ for every $A \in \mathscr{B}(E)$. The Radon measure $\mu$ is called a convex Radon measure if it satisfies that $1=\sup \{\mu(K) ; K$ is compact and convex\}. If $E$ is quasi-complete, then every Radon measure is convex Radon since the closed convex hull of each compact subset is again compact.

The weakest notion of the $0-1$ law is the following.

(0) For every $x^{\prime} \in E^{\prime}, \mu\left(x ; x^{\prime}(x)=0\right)=0$ or 1 .

The strongest $0-1$ law is the following.

(1) For every $\mu$-measurable linear subspace $F \subset E, \mu(F)=0$ or 1 .

Communicated by S. Matsuura, July 11, 1985.

* Department of Mathematics, Yamaguchi University, Yamaguchi 753, Japan.

** Department of Mathematics, Kyushu University. Fukuoka 812. Japan. 
For example, convex measures, semi-stable measures satisfy the $0-1$ law (1), see Borell [1], Dudley and Kanter [2], Krakowiak [4] and Louie, Rajput and Tortrat [5].

We introduce other intermediate $0-1$ laws between $(0)$ and (1). Let $l_{\infty}=$ $\left\{\left(t_{n}\right) \in \boldsymbol{R}^{\infty} ; \sup _{n}\left|t_{n}\right|<\infty\right\}, l_{p}=\left\{\left(t_{n}\right) \in \boldsymbol{R}^{\infty} ; \Sigma_{n}\left|t_{n}\right|^{p}<\infty\right\} \quad(1 \leqq p<\infty), \quad c_{1}=\left\{\left(t_{n}\right) \in \boldsymbol{R}^{\infty} ;\right.$ $\lim _{n} t_{n}$ exists $\}$ and $c_{0}=\left\{\left(t_{n}\right) \in \boldsymbol{R}^{\infty} ; t_{n} \rightarrow 0\right\}$ be the usual Banach spaces, where $\boldsymbol{R}^{\infty}$ be the countable product of the real numbers $\boldsymbol{R}$ with the product topology. A linear subspace $F$ of $\boldsymbol{R}^{\infty}$ is called a convex Lusin subspace if $F$ is a Borel subset of $\boldsymbol{R}^{\infty}$ and, for every probability measure $\nu$ on $\boldsymbol{R}^{\infty}$, it holds that $\nu(F)=$ $\sup \left\{\nu(K) ; K \subset F, K\right.$ is compact convex and balanced in $\left.\boldsymbol{R}^{\infty}\right\}$. For example, $l_{\infty}$, $l_{p}(1 \leqq p<\infty), \quad c_{1}$ and $c_{0}$ are convex Lusin subspaces of $\boldsymbol{R}^{\infty}$. Every separable Banach subspace is convex Lusin.

(2) For every sequence $x_{n}^{\prime} \in E^{\prime}$ and every convex Lusin subspace $F$ of $\boldsymbol{R}^{\infty}$, $\mu\left(x ;\left(x_{n}^{\prime}(x)\right) \in F\right)=0$ or 1 .

(3) For every sequence $x_{n}^{\prime} \in E^{\prime}, \mu\left(x ;\left(x_{n}^{\prime}(x)\right) \in l_{\infty}\right)=0$ or 1 .

(4) For every sequence $x_{n}^{\prime} \in E^{\prime}, \mu\left(x ;\left(x_{n}^{\prime}(x)\right) \in c_{1}\right)=0$ or 1 .

(5) For every sequence $x_{n}^{\prime} \in E^{\prime}, \mu\left(x ;\left(x_{n}^{\prime}(x)\right) \in c_{0}\right)=0$ or 1 .

(6) There exist no sequence $x_{n}^{\prime} \in E^{\prime}$ such that $\mu\left(x ;\left(x_{n}^{\prime}(x)\right) \in c_{0}\right)>0$ and that $\mu\left(x ;\left(x_{n}^{\prime}(x)\right) \notin l_{\infty}\right)>0$.

The 0-1 law (3) was considered by Sato [6]. It is clear that the $0-1$ law (2) is stronger than (3), (4) and (5). It is also clear that each of (3), (4) and (5) is stronger than (6).

The following 0-1 law (7) is weaker than (5).

(7) For every sequence $x_{n}^{\prime} \in E^{\prime}$, if there exists $\left(a_{n}\right) \in c_{0}$ such that $\mu\left(x ;\left(a_{n}^{-1} x_{n}^{\prime}(x)\right) \in l_{\infty}\right)>0$, then $\mu\left(x ;\left(x_{n}^{\prime}(x)\right) \in c_{0}\right)=1$.

In fact, if $\left(a_{n}^{-1} x_{n}^{\prime}(x)\right) \in l_{\infty}$ for $\left(a_{n}\right) \in c_{0}$, then $\left(x_{n}^{\prime}(x)\right) \in c_{0}$. Hence $(5) \Rightarrow(7)$ follows.

(8) For every sequence $x_{n}^{\prime} \in E^{\prime}$, if there exists $\left(a_{n}\right) \in c_{0}$ such that $\mu\left(x ;\left(a_{n}^{-1} x_{n}^{\prime}(x)\right) \in l_{\infty}\right)>0$, then $\mu\left(x ;\left(x_{n}^{\prime}(x) \in l_{\infty}\right)=1\right.$.

(9) For every sequence $x_{n}^{\prime} \in E^{\prime}$, if there exists $\left(a_{n}\right) \in c_{0}$ such that $\mu\left(x ;\left(a_{n}^{-1} x_{n}^{\prime}(x)\right) \in c_{1}\right)>0$, then $\mu\left(x ;\left(x_{n}^{\prime}(x)\right) \in c_{1}\right)=1$.

(10) For every sequence $x_{n}^{\prime} \in E^{\prime}$, if there exists $\left(a_{n}\right) \in c_{0}$ such that $\mu\left(x ;\left(a_{n}^{-1} x_{n}^{\prime}(x)\right) \in c_{0}\right)>0$, then $\mu\left(x ;\left(x_{n}^{\prime}(x)\right) \in c_{0}\right)=1$. 
(11) For every sequence $x_{n}^{\prime} \in E^{\prime}$, if there exists $\left(a_{n}\right) \in c_{0}$ such that $\mu\left(x ;\left(a_{n}^{-1} x_{n}^{\prime}(x)\right) \in c_{0}\right)>0$, then $\mu\left(x ;\left(x_{n}^{\prime}(x)\right) \in l_{\infty}\right)=1$.

Since $c_{0} \subset c_{1} \subset l_{\infty}$, it is easily seen that the $0-1$ law (7) is stronger than (8), (9) and (10). Also each of (8), (9) and (10) is stronger than (11). It is clear that the 0-1 law (6) implies (8) and (11), since if $\mu\left(x ;\left(a_{n}^{-1} x_{n}^{\prime}(x)\right) \in l_{\infty}\right)>0$, then $\mu\left(x ;\left(x_{n}^{\prime}(x)\right) \in c_{0}\right) \geqq \mu\left(x ;\left(a_{n}^{-1} x_{n}^{\prime}(x)\right) \in l_{\infty}\right)>0$ and hence by $(6), \mu\left(x ;\left(x_{n}^{\prime}(x)\right) \in l_{\infty}\right)=1$. We shall show that the 0-1 law (11) implies (2). Thus we obtain the main result (Theorem 1):

The $0-1$ laws (2) (11) are all equivalent.

Since $l_{p}(1 \leqq p<\infty)$ is a convex Lusin subspace of $\mathbb{R}^{\infty}$, each of $(2) \sim(11)$ implies the following $0-1$ law.

(12) For every sequence $x_{n}^{\prime} \in E^{\prime}, \mu\left(x ;\left(x_{n}^{\prime}(x)\right) \in l_{p}\right)=0$ or $1(1 \leqq p<\infty)$.

The above $0-1$ laws $(2) \sim(12)$ are described in terms of the sequences in $E^{\prime}$, so it is sufficient to suppose that the measure $\mu$ is defined only on $C\left(E, E^{\prime}\right)$. We state another $0-1$ laws for a Radon probability measure $\mu$ on $\mathcal{B}(E)$.

(13) For every closed convex balanced subset $B, \mu\left(\bigcup_{n=1}^{\infty} n B\right)=0$ or 1 .

(14) For every lower semi-continuous semi-norm $N(x)$ on $E$ (admitting the value $\infty), \mu(x ; N(x)<\infty)=0$ or 1 .

(15) For every compact convex balanced subset $K, \mu\left(\bigcup_{n=1}^{\infty} n K\right)=0$ or 1 .

For example, the countable product of non-atomic probability measures on $\mathbb{R}^{\infty}$ satisfies the 0-1 law (15), see Hoffmann-Jørgensen [3] and Zinn [8]. Obviously (13) and (14) are equivalent. We show that if $\mu$ is Radon, then (2) (11), (13) and (14) are equivalent (Theorem 2). In the case where $\mu$ is convex Radon, (13) and (15) are equivalent, hence the $0-1$ laws (2) (11), (13) (14) and (15) are all equivalent (Theorem 3). The implication $(3) \Rightarrow(15)$ for a convex Radon $\mu$ was proved by Sato [6] and $(15) \Rightarrow(3)$ was remarked by Takahashi [7].

\section{§2. Main Results}

Theorem 1. The 0-1 laws (2) (11) are all equivalent.

Proof. As we have remarked in the preceding section, it is sufficient to prove $(11) \Rightarrow(2)$. Let $x_{n}^{\prime} \in E^{\prime}$ and $F$ be a convex Lusin subspace of $\mathbb{R}^{\infty}$. Suppose that $\mu\left(x ;\left(x_{n}^{\prime}(x)\right) \in F\right)>0$. Then we must show that $\mu\left(x:\left(x_{n}^{\prime}(x)\right) \in F\right)=1$. If we 
set $\Pi: E \rightarrow \boldsymbol{R}^{\infty}$ by $\Pi(x)=\left(x_{n}^{\prime}(x)\right)$, then by the definition of the convex Lusin subspace, there exists a compact convex balanced subset $K$ in $\boldsymbol{R}^{\infty}$ such that $K \subset F$ and that $\Pi(\mu)(K)=\mu\left(\Pi^{-1}(K)\right)>0$, where $\Pi(\mu)$ is the image measure of $\mu$ by $\Pi$. Let $\bigcup_{n=1}^{\infty} n K$ be the linear subspace of $\boldsymbol{R}^{\infty}$ spanned by $K$. We show $\Pi(\mu)\left(\bigcup_{n=1}^{\infty} n K\right)=1$.

Suppose that $\Pi(\mu)\left(\bigcup_{n=1}^{\infty} n K\right)<1$. Then there exists a compact subset $L$ in $\boldsymbol{R}^{\infty}$ such that

$$
\Pi(\mu)(L)>0 \text { and } L \cap\left(\bigcup_{n=1}^{\infty} n K\right)=\varnothing .
$$

For every $x \in L$ and every $n$, by the Hahn-Banach theorem, there exists $\xi_{n, x} \in\left(\boldsymbol{R}^{\infty}\right)^{\prime}$ such that

$$
\xi_{n, x}(x)>1 \text {, and }\left|\xi_{n, x}(y)\right| \leqq 1 \text { on } n K \text {, }
$$

that is, $\xi_{n, x}$ separates $n K$ and $x$. For every fixed $n$, the open subsets $U_{n, x}=$ $\left\{y: \xi_{n, x}(y)>1\right\}, x \in L$, form a covering of $L$. Take a finite sub-covering $U_{n, x_{j}^{n}}$, $j=1,2, \cdots, j(n)$. Then for every $n$ and every $x \in L$, there exists some $j \in\{1,2, \cdots, j(n)\}$ such that

$$
\xi_{n, x_{j}^{n}}(x)>1
$$

Consider the following sequence in $\left(\boldsymbol{R}^{\infty}\right)^{\prime}$ :

$\left.{ }^{*}\right) \quad \xi_{1, x_{1}^{1}}, \cdots, \xi_{1, x_{j(1)}^{1}}, \cdots, n^{1 / 3} \xi_{n, x_{1}^{n}}, \cdots, n^{1 / 3} \xi_{n, x_{j(n)}^{n}},(n+1)^{1 / 3} \xi_{n+1, x_{1}^{n+1}}, \cdots$.

Then for every $x \in L$ and every $n$, there exists suitable $j$ in $\{1,2, \cdots, j(n)\}$ such that

$$
n^{1 / 3} \xi_{n, x_{j}^{n}}(x)>n^{1 / 3} .
$$

On the other hand, for every $x \in m K$ ( $m$ be arbitrarily fixed), we have as $n \rightarrow \infty$,

$$
\begin{aligned}
n^{2 / 3} \xi_{n, x_{j}^{n}}(x) & =n^{2 / 3} \frac{m}{n} \xi_{n, x_{j}^{n}}\left(\frac{n}{m} x\right) \\
& \leqq n^{2 / 3} \frac{m}{n}=m n^{-1 / 3} \rightarrow 0,
\end{aligned}
$$

since $(n / m) x \in n K$. Thus we have

$$
n^{2 / 3} \xi_{n, x_{j}}(x) \rightarrow 0 \text { on } \bigcup_{m=1}^{\infty} m K,
$$

as $n \rightarrow \infty$. Denote by $\left(\eta_{k}\right)$ the sequence $(*)$ and $\left(a_{k}\right) \in c_{0}$ be the following sequence :

that is,

$$
1, \cdots, 1, \cdots, n^{1 / 3}, \cdots, n^{1 / 3},(n+1)^{1 / 3}, \cdots
$$

$$
a_{k}=n^{1 / 3} \quad \text { for } \quad \sum_{i=1}^{n-1} j(i)<k \leqq \sum_{i=1}^{n} j(i)
$$


We put $z_{k}^{\prime}=\eta_{k} \circ \Pi$. Then $z_{k}^{\prime} \in E^{\prime}$ and it hold that

$$
\begin{aligned}
& a_{k} z_{k}^{\prime}(x) \rightarrow 0 \quad \text { on } \Pi^{-1}\left(\bigcup_{m=1}^{\infty} m K\right), \quad \text { and } \\
& \sup _{k}\left|z_{k}^{\prime}(x)\right|=\infty \text { on } \Pi^{-1}(L),
\end{aligned}
$$

which contradict to (11), since $\mu\left(\Pi^{-1}\left(\bigcup_{m=1}^{\infty} m K\right)\right)>0$ and $\mu\left(\Pi^{-1}(L)\right)>0$.

This completes the proof.

Corollary 1. Each of the 0-1 law (2) (11) implies (12).

Theorem 2. Suppose that $\mu$ is a Radon probability measure. Then the $0-1$ laws (2) (11), (13) and (14) are all equivalent.

Proof. By Theorem 1, it is sufficient to show $(5) \Leftrightarrow(13)$.

(5) $\Rightarrow(13)$ Let $B$ be a closed convex balanced subset with $\mu(B)>0$. We show that $\mu\left(\bigcup_{n=1}^{\infty} n B\right)=1$. Suppose that $\mu\left(\bigcup_{n=1}^{\infty} n B\right)<1$. Then since $\mu$ is Radon, there is a compact subset $L$ such that

$$
\mu(L)>0 \text { and } L \cap\left(\bigcup_{n=1}^{\infty} n B\right)=\varnothing .
$$

For every $n$ and every $x \in L$, by the Hahn-Banach theorem, there exists $\xi_{n, x} \in E^{\prime}$ such that

$$
\xi_{n, x}(x)>1 \text {, and }\left|\xi_{n, x}(y)\right| \leqq 1 \text { on } n B \text {. }
$$

The open subsets $U_{n, x}=\left\{y, \xi_{n, x}(y)>1\right\}, x \in L$, cover $L$. Take a finite subcovering $U_{n, x_{j}^{n}}, j=1,2, \cdots, j(n)$ and consider the sequence

$$
\xi_{1, x_{1}^{1}}, \cdots, \xi_{1, x_{j(1)}^{1}}, \cdots, \xi_{n, x_{1}^{n}}, \cdots, \xi_{n, x_{j(n)}^{n}}, \cdots .
$$

Then we have

$$
\sup _{n}\left|\xi_{n, x_{j}^{n}}(x)\right|>1 \text { on } L \text {, and } \xi_{n, x_{j}^{n}}(x) \rightarrow 0 \text { on } \bigcup_{m=1}^{\infty} m B
$$

since for every $x \in m B$

$$
\begin{aligned}
\xi_{n, x_{j}^{n}}(x) & =\frac{m}{n} \xi_{n, x_{j}^{n}}\left(\frac{n}{m} x\right) \\
& \leqq \frac{m}{n} \rightarrow 0 \text { as } n \rightarrow \infty
\end{aligned}
$$

These contradict to (5).

$(13) \Rightarrow(5)$ Suppose that $\mu\left(x ;\left(x_{n}^{\prime}(x)\right) \in c_{0}\right)>0$, that is, $\mu\left(x ; x_{n}^{\prime}(x) \rightarrow 0\right)>0$. By the Egorov's theorem, there exists $A \subset\left\{x ; x_{n}^{\prime}(x) \rightarrow 0\right\}$ such that $\mu(A)>0$ and $x_{n}^{\prime}(x) \rightarrow 0$ uniformly on $A$. Let $B$ be the closed convex balanced hull of $A$. We shall see $x_{n}^{\prime}(x) \rightarrow 0$ uniformly also on $B$. For every $\varepsilon>0$, there is an $N=N(\varepsilon)$ 
such that

$$
\sup _{x \in A}\left|x_{n}^{\prime}(x)\right|<\varepsilon \quad \text { for every } n>N
$$

since $\left(x_{n}^{\prime}\right)$ converges to 0 uniformly on $A$. The subset $\left\{x ;\left|x_{n}^{\prime}(x)\right| \leqq \varepsilon\right.$ for every $n>N\}$ is closed convex balanced, and contains $A$ so it follows that $B \subset\left\{x ;\left|x_{n}^{\prime}(x)\right| \leqq \varepsilon\right.$ for every $\left.n>N\right\}$.

Hence we have

$$
\sup _{x \in B}\left|x_{n}^{\prime}(x)\right| \leqq \varepsilon \quad \text { for every } \quad n>N,
$$

which proves the assertion. By the assumption (13), we have

$$
\mu\left(x ; x_{n}^{\prime}(x) \rightarrow 0\right) \geqq \mu\left(\bigcup_{n=1}^{\infty} n B\right)=1 .
$$

Thus the $0-1$ law (5) is valid.

This completes the proof.

Theorem 3. If $\mu$ is a convex Radon measure, then the $0-1$ laws $(2) \sim(11)$, (13), (14) and (15) are all equivalent.

Proof. It is sufficient to show $(13) \Leftrightarrow(15) . \quad(13) \Rightarrow(15)$ is obvious.

$(15) \Rightarrow(13)$ Let $B$ be a closed convex balanced subset with $\mu(B)>0$. We show that $\mu\left(\bigcup_{n=1}^{\infty} n B\right)=1$. Since $\mu$ is a convex Radon measure, there exists a compact convex balanced subset $K$ such that $\mu(K \cap B)>0$. Let $L$ be the closed convex balanced hull of $K \cap B$. Since $L$ is a closed subset of the compact set $K, L$ is also compact. By the assumption (15), we have $\mu\left(\bigcup_{n=1}^{\infty} n B\right) \geqq \mu\left(\bigcup_{n=1}^{\infty} n L\right)$ $=1$.

This completes the proof.

\section{References}

[1] Borell, C., Convex measures on locally convex spaces, Ark. för Math., 12 (1974), 239-252.

[2] Dudley, R.M. and Kanter, M., Zero-one laws for stable measures, Proc. A.M.S., 45 (1974), 245-252.

[3] Hoffmann-Jørgensen, J., Integrability of seminorms, the 0-1 law and affine kernel for product measures, Studia Math., 61 (1977), 137-159.

[4] Krakowiak, W., Zero-one laws for stable and semi-stable measures on Banach spaces, Bull. Acad. Sci. Polonaise, 27 (1978), 1045-1049.

[5] Louie, D., Rajput, B. and Tortrat, A., A zero-one dichotomy theorem for r-semistable laws on infinite-dimensional linear spaces, Sankya Ser. A, 42 (1980), 9-18.

[6] Sato, H., Banach support of a probability measure in a locally convex space, Lecture Notes in Math., 526, Springer.

[7] Takahashi, Y., Hilbertian support of probability measures on locally convex spaces and their applications, Hokkaido Math. J., 10 (1981), 57-74.

[8] Zinn, J., Zero-one laws for non-Gaussian measures, Proc. A.M.S., 44 (1974), 179 185. 